\title{
Precision radial velocities of double-lined binary stars and the spectroscopic follow-up of circumbinary transiting planet candidates
}

\author{
Maciej Konacki \\ Nicolaus Copernicus Astronomical Center, Polish Academy of Sciences, \\ Rabianska 8, 87-100 Torun, Poland \\ Astronomical Observatory, A. Mickiewicz University, \\ Sloneczna 36, 60-286 Poznan, Poland \\ email: maciej@ncac.torun.pl
}

\begin{abstract}
We present a new iodine cell based approach that allows one to obtain radial velocities of the components of double-lined spectroscopic binary stars (SB2s) with a precision reaching $5 \mathrm{~m} / \mathrm{s}$. Such an RV precision is up to 100 times better than what is currently available in the literature for the SB2s. We discuss the applications of our method to the radial velocity searches for circumbinary planets and spectroscopic follow-up of transiting planet candidates around eclipsing binary stars.
\end{abstract}

\section{Introduction}

The precision radial velocity (RV) technique for single stars is the driving force in the extrasolar planets field. It has been used to detect most of the known extrasolar planets as well as confirm and characterize transiting planet candidates from photometric surveys. Binary (and multiple) stars were traditionally omitted from the surveys due to problems with obtaining high RV precision for their components. In particular, the original iodine absorption cell approach was not designed to measure RVs of binary stars. Yet, more than half the stars from the solar neighborhood are in binary or multiple star systems.

It is tempting to find a way to precisely measure RVs of binary stars not only for extrasolar planets studies but also for general stellar astronomy. To this end, we developed a modification of the iodine cell technique which can handle spectra of spectroscopic binaries (Konacki 2005). Using the Keck I/HIRES spectrograph, we demonstrated an RV precision of 20-30 m/s for binary stars. Since that time, we have modified this approach by introducing changes to the numerical side of the method and substantially modifying the method itself. Now, the method can in favorable cases produce an RV precision as high as $5 \mathrm{~m} / \mathrm{s}$ for the components of double-lined spectroscopic binary stars (SB2s).

Below, we briefly describe the new approach as well as its tests based on a sample of SB2s observed with Keck I/HIRES over the period 2003-2007. We also discuss its applications to the RV searches for circumbinary planets and spectroscopic follow-up of transiting planet candidates around eclipsing binary stars.

\section{Iodine cell technique and binary stars}

In the iodine cell $\left(\mathrm{I}_{2}\right)$ technique, the Doppler shift of a star spectrum $\Delta \lambda$ is determined by solving the following equation (Marcy \& Butler 1992).

$$
I_{o b s}(\lambda)=\left[I_{s}\left(\lambda+\Delta \lambda_{s}\right) T_{I_{2}}\left(\lambda+\Delta \lambda_{I_{2}}\right)\right] \otimes P S F
$$




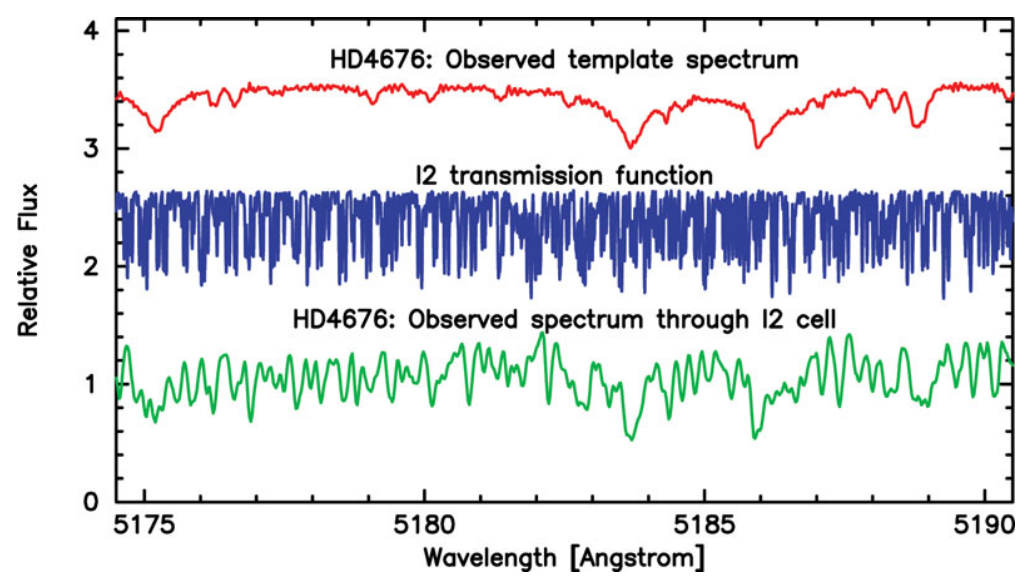

Figure 1. A typical observing sequence for the iodine cell based approach to the precision RVs. A stellar spectrum is collected without (top) and with (bottom) the iodine cell. The spectrum taken with the iodine cell is then modeled using a high resolution $\left(\mathrm{R} \approx 10^{6}\right)$ iodine cell transmission function (middle) to obtain the wavelength solution and the spectrograph's PSF. The figure covers only a fraction of the entire iodine spectrum, which spans 5000-6200.

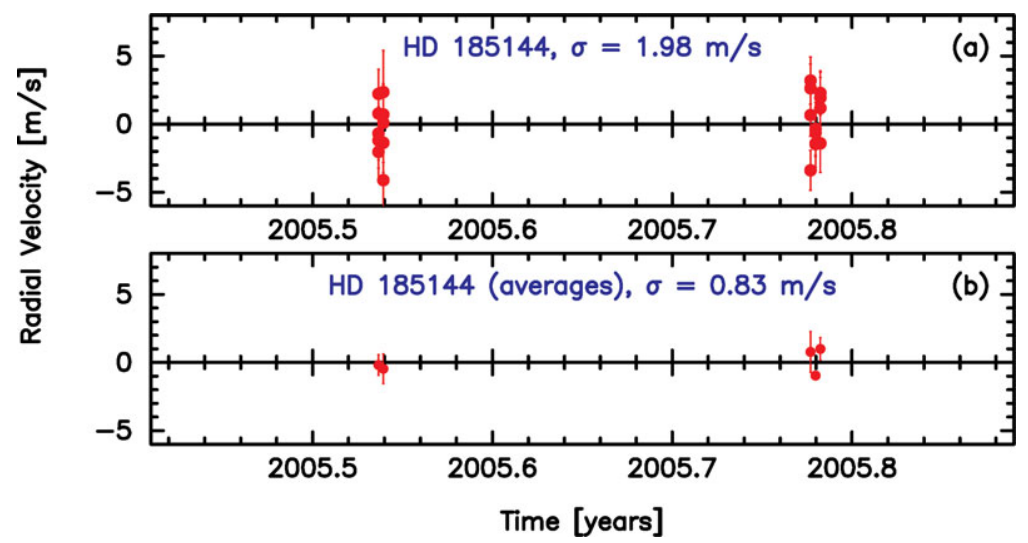

Figure 2. A series of precision RV measurements of a single star HD185144 ( $\mathrm{V}=4.7 \mathrm{mag}$, K0V) taken with the Keck I/HIRES and reduced using our new iodine cell data pipeline. The data were taken on 5 nights: two in July ( 5 consecutive spectra each night) and three in October 2005 (4 consecutive spectra each night). The average SNR was 600 for the template exposure as well as for the spectra taken with the iodine cell.

where $\Delta \lambda_{s}$ is the shift of the star spectrum, $\Delta \lambda_{I_{2}}$ is the shift of the iodine transmission function $T_{I_{2}}, \otimes$ represents a convolution and $P S F$ a spectrograph's point spread function (PSF). The parameters $\Delta \lambda_{s}, \Delta \lambda_{I_{2}}$ as well as parameters describing the PSF are determined by performing a least-squares fit to the spectrum observed through the iodine cell, $I_{o b s}$. For this purpose, one also needs (1) a high-SNR star spectrum taken without the cell, $I_{s}$, which serves as a template for all the spectra observed through the cell and (2) the $I_{2}$ transmission function $T_{I_{2}}$ obtained with the Fourier Transform Spectrometer at the Kitt Peak National Observatory. The Doppler shift of a star spectrum is then given by $\Delta \lambda=\Delta \lambda_{s}-\Delta \lambda_{I_{2}}$. Such an iodine technique can only be applied to single stars. This is dictated by the need to supply an observed template spectrum of each component of a target binary star in Eq. 2.1. In the case of SB2s, it cannot be accomplished since their spectra are always composite and time variable. 


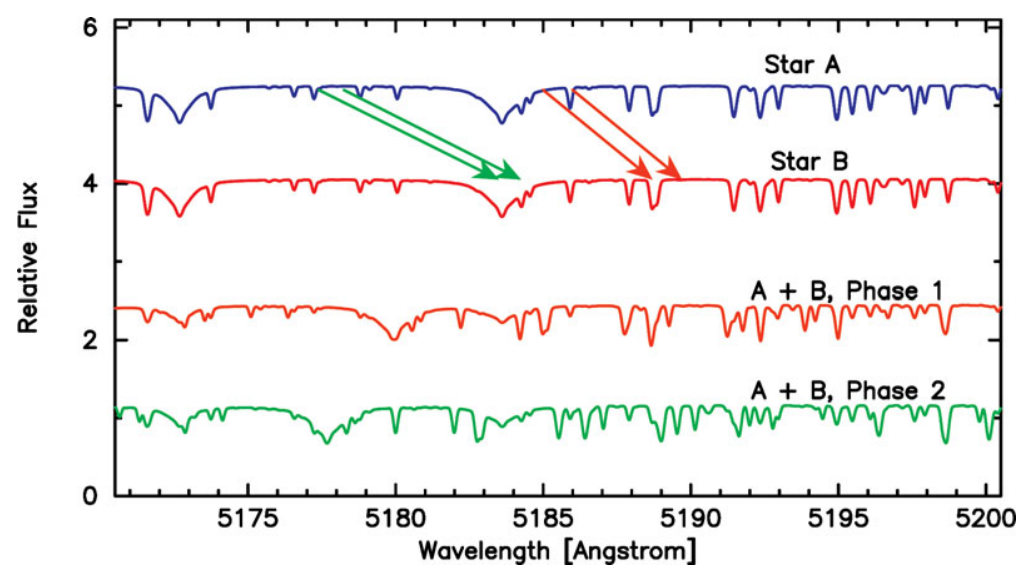

Figure 3. An SB2 as Nature's realization of tomographic imaging. The composite spectrum of an SB2 taken at any orbital phase can be interpreted as a result of the imaging of a two-layered object. By observing an SB2 at several different orbital phases and hence different RVs of its components, one can carry out an equivalent of tomographic imaging and eventually be able to disentangle the component ("layer") spectra.

We can measure precise radial velocities of both components of an SB2 with an $\mathrm{I}_{2}$ absorption cell by performing the following steps. First, contrary to the standard approach for single stars, we always take two subsequent exposures of a binary target - one with and the other without the $\mathrm{I}_{2}$ cell (see Fig. 1). This way we obtain an instantaneous template which is used to model only the adjacent exposure taken with the cell. Next, we perform the usual least-squares fit and obtain the parameters described in Eq. 2.1. Obviously, the derived Doppler shift, $\Delta \lambda_{i}$ (where $i$ denotes the epoch of the observation), carries no meaning since each time a different template is used (besides it describes a Doppler "shift" of a composite spectrum that is typically different at each epoch). However, the parameters (in particular the wavelength solution and the parameters describing PSF) are accurately determined and can be used to extract the star spectrum, $I_{o b s}^{\star, i}(\lambda)$, for each epoch $i$, by inverting equation 2.1

$$
I_{o b s}^{\star, i}(\lambda)=\left[I_{o b s}^{i}(\lambda) \otimes^{-1} P S F^{i}\right] / T_{I_{2}}(\lambda)
$$

where $\otimes^{-1}$ denotes deconvolution and $P S F^{i}$, symbolically, the set of parameters describing PSF at the epoch $i$. Such a star spectrum has an accurate wavelength solution, is free of the $\mathrm{I}_{2}$ lines and the influence of a varying PSF. In the final step, the velocities of both components of a binary target can be measured with the two-dimensional crosscorrelation technique TODCOR (Zucker \& Mazeh 1994) using as templates the synthetic spectra derived with the ATLAS 9 and ATLAS 12 programs (Kurucz 1995) and matched to the observed spectrum, $I_{s}(\lambda)$.

\section{Improvements and tests}

The above approach allowed us to obtain an RV precision of $20-30 \mathrm{~m} / \mathrm{s}$ for binary stars (Konacki 2005). Such a precision is sufficient to search for Jupiter-mass shortperiod planets. Although such a precision is dozens of times better than that of the RV data sets for spectroscopic binary stars available in the literature, it is also at least ten times worse than the current state-of-the-art RV precision for single stars. Since our spectroscopic data for binaries satisfy all the quality requirements and are taken with the 


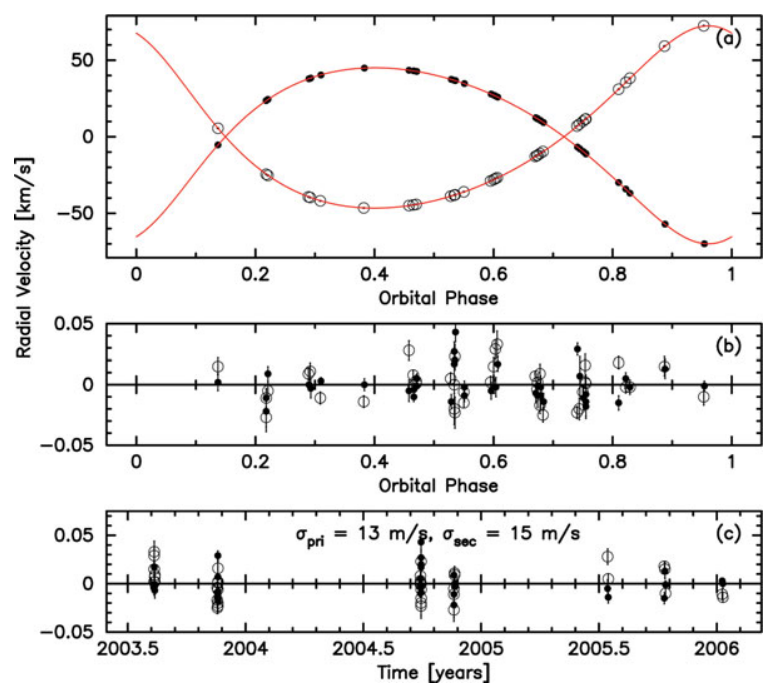

Figure 4. The RVs of HD4676 taken with the Keck I/HIRES as a function of the orbital phase (a), and the residuals (observed minus modeled RVs) as a function of the orbital phase (b) and time (c). The primary is denoted with filled circles, the secondary with open ones and the best-fit RV model with a solid line. HD4676 is a bright binary $(\mathrm{V}=5.1 \mathrm{mag})$ composed of two F6V stars (the brightness ratio is 1). It has an orbital period of 13.8 days, eccentricity 0.23 and RV semi-amplitudes of $57.5 \mathrm{~km} / \mathrm{s}$ and $59.5 \mathrm{~km} / \mathrm{s}$ for the primary and the secondary respectively. The time span of the data is 2.5 years and includes $82 \mathrm{RV}$ measurements (41 for each component). The average SNR for each spectrum is about 250 which corresponds to an SNR of 125 for the primary and secondary. The best-fit RV rms is $13 \mathrm{~m} / \mathrm{s}$ for the primary and $15 \mathrm{~m} / \mathrm{s}$ for the secondary.

same equipment as those of single stars, it seemed that there must be a way to measure RVs of spectroscopic binary stars with a precision similar to that of single stars.

In order to improve the precision, we first decided to work on the numerical aspects of our data pipeline. This was motivated by equation 2.2 that involves the deconvolution of a numerically determined PSF followed by a removal of the iodine lines. It is a step which is not done for single stars and presumably could be a potential source of systematic RV errors. This task has evolved into creating a completely new iodine data pipeline, which now for single stars produces RV precisions below $1 \mathrm{~m} / \mathrm{s}$ (see Fig. 2). Surprisingly, this has not improved the RV precision for binary stars.

The other suspect for a lower RV precision is the use of synthetic template spectra and/or TODCOR. First, even the best synthetic template spectra are never an exact match for the components' spectra of a binary. This most certainly affects the final RV precision. Second, TODCOR, while being a very well tested and reliable technique, has never been tested at $\mathrm{m} / \mathrm{s}$ precision. To overcome these two problems, we decided to disentangle the composite spectra of our binaries to produce the real (observed) component spectra.

Disentangling the spectra of SB2s is a well-known problem to which several more or less restricted solutions exist and are described in a rich literature. We decided to follow the tomographic approach to the disentangling problem by Bagnuolo \& Gies (1991) (see Fig. 3) which we have numerically solved using our own variation of the tomographic set of equations and employing the maximum-entropy method performed with the commercially available package MEMSYS5 (for full details see Konacki 2008, in preparation). Note that once one has the real template spectra for the components of a binary, it is no 


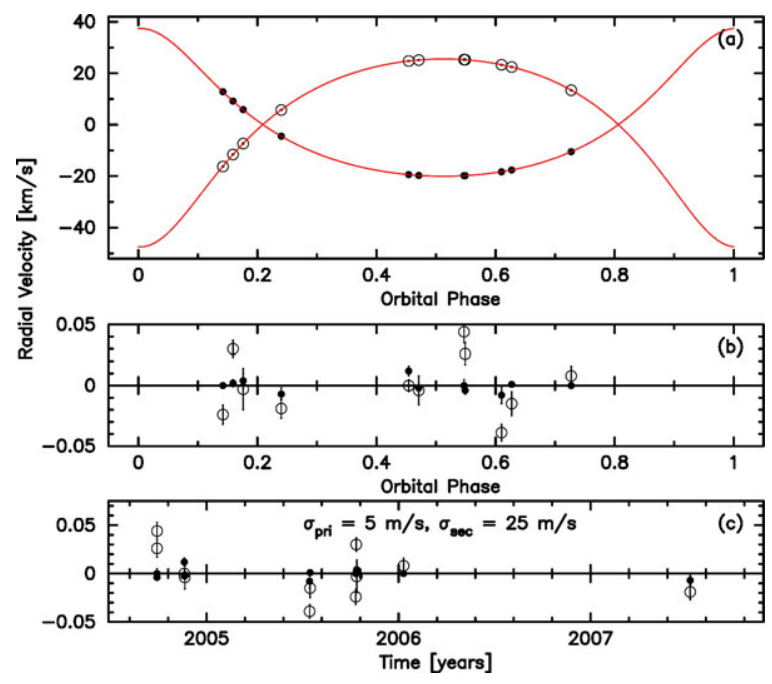

Figure 5. The RVs of HD195987 taken with the Keck I/HIRES as a function of the orbital phase (a), and the residuals (observed minus modeled RVs) as a function of the orbital phase (b) and time (c). The primary is denoted with filled circles, the secondary with open ones and the best-fit RV model with a solid line. HD195987 is a bright binary ( $\mathrm{V}=7.1 \mathrm{mag}$ ) composed of G0V and $\mathrm{K} 3 \mathrm{~V}$ stars (the brightness ratio is 6.7 ). It has an orbital period of 57.3 days, eccentricity 0.3 and the RV semi-amplitudes of $28.7 \mathrm{~km} / \mathrm{s}$ and $36.5 \mathrm{~km} / \mathrm{s}$ for the primary and the secondary respectively. The time span of the data is 2.8 years and includes $22 \mathrm{RV}$ measurements (11 for each component). The average SNR for each spectrum is about 250 which corresponds to an SNR of 220 for the primary and 30 for the secondary. The best-fit RV rms is $5 \mathrm{~m} / \mathrm{s}$ for the primary and $25 \mathrm{~m} / \mathrm{s}$ for the secondary.

longer necessary to use TODCOR as one can simply use real templates and the original iodine equation 2.1 by replacing $I_{s}(\lambda+\Delta \lambda)$ with $I_{s}^{1}\left(\lambda+\Delta \lambda_{s}^{1}\right)+I_{s}^{2}\left(\lambda+\Delta \lambda_{s}^{2}\right)$, where $I_{s}^{1}, I_{s}^{2}$ represent the two templates and $\Delta \lambda_{s}^{1}, \Delta \lambda_{s}^{2}$ their shifts. Still, TODCOR remains an important intermediate step to get the first approximate RVs that are subsequently used in the disentangling scheme.

This approach has turned out to be a very successful one as is demonstrated in Figures 4 to 6 . In particular, one can see that if a binary star satisfies typical requirements - a sufficiently late spectral type, low $v \sin i$, low level of activity - then a nearly photon limited RV precision can be obtained (Fig. 5). Note that for a binary star, the SNR of a component's spectrum will depend on the SNR of the composite observed spectrum and the brightness ratio of the two stars. Hence, the RV precision for the fainter secondary may be substantially lower than for the primary if the brightness ratio is large.

\section{Applications}

The first straightforward application of our method is an RV search for circumbinary planets. To this end, in 2003 we have initiated a pilot survey of 12 SB2s with Keck I/HIRES (until mid 2007). This survey was relocated for a brief period to TNG/SARG (2006-2007) and since late 2006 is carried out in collaboration with Matt Muterspaugh using the Hamspec spectrograph and the Shane and CAT telescopes of the Lick Observatory. Our current sample consists of about 40 mostly northern hemisphere, predominantly non-eclipsing SB2s and is increasing. The improvement in the precision now enables us to reach the planetary mass limit similar to that of extrasolar planets around single stars (see Figs. 7-8, solid lines). Obviously after taking into account the lower limit for 


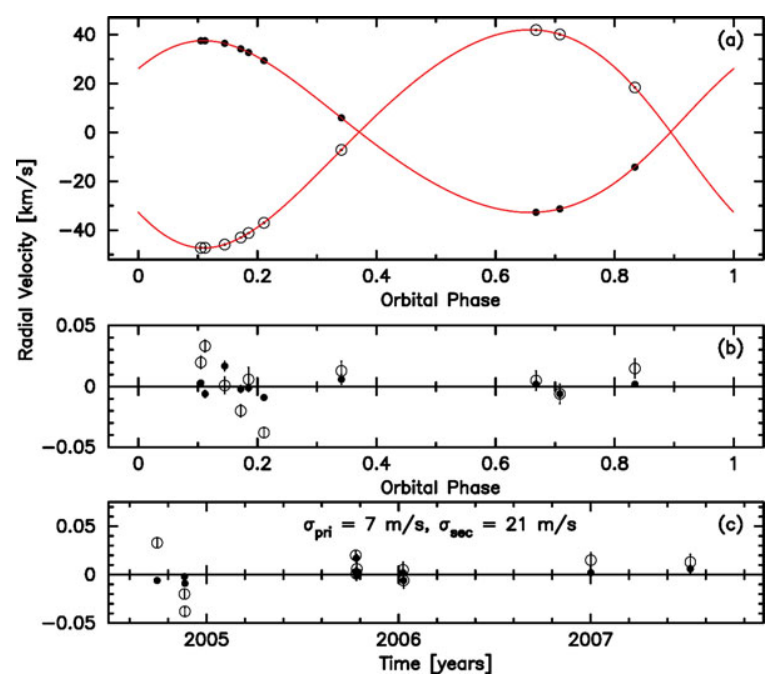

Figure 6. The RVs of HD9939 taken with Keck I/HIRES as a function of orbital phase (a), and the residuals (observed minus modeled RVs) as a function of orbital phase (b) and time (c). The primary is denoted with filled circles, the secondary with open circles and the best-fit RV model with a solid line. HD9939 is a bright binary ( $=7.0 \mathrm{mag}$ ) composed of two K0IV stars (the brightness ratio is 6.3). It has an orbital period of 25.2 days, eccentricity 0.1 and RV semi-amplitudes of $35.1 \mathrm{~km} / \mathrm{s}$ and $44.6 \mathrm{~km} / \mathrm{s}$ for the primary and the secondary respectively. The time span of the data is 2.7 years and includes $20 \mathrm{RV}$ measurements (10 for each component). The average SNR for each spectrum is about 250, which corresponds to a SNR of 215 for the primary and 35 for the secondary. The best-fit RV rms is $7 \mathrm{~m} / \mathrm{s}$ for the primary and $21 \mathrm{~m} / \mathrm{s}$ for the secondary.

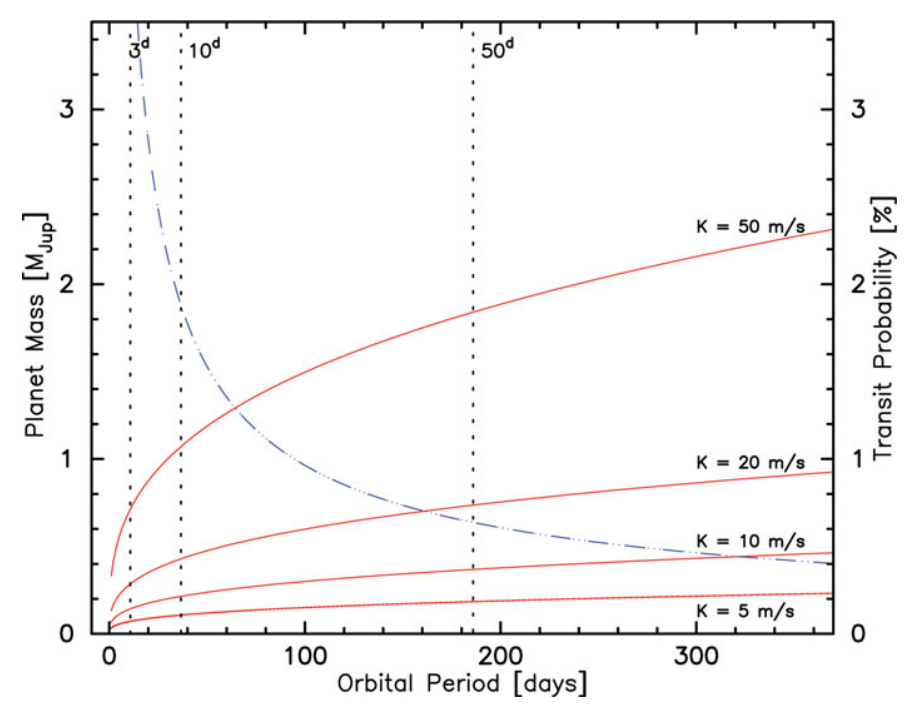

Figure 7. The discovery space (orbital period vs mass) for a putative circumbinary planet around an eclipsing binary composed of 1.0 and $0.5 \mathrm{M}_{\odot}$ stars. The solid (red) lines denote the planets corresponding to central body's RV amplitudes of 5, 10, 20 and $50 \mathrm{~m} / \mathrm{s}$. The dashed (blue) line denotes the geometric transit probability assuming that the "effective radius" of an eclipsing binary is $1 \mathrm{R}_{\odot}$. The dotted vertical lines denote the limits for a dynamically stable circumbinary planet given the orbital period (3,10 and 50 days) of the inner stellar binary. All planetary orbits to the right of the dotted line are dynamically stable. 


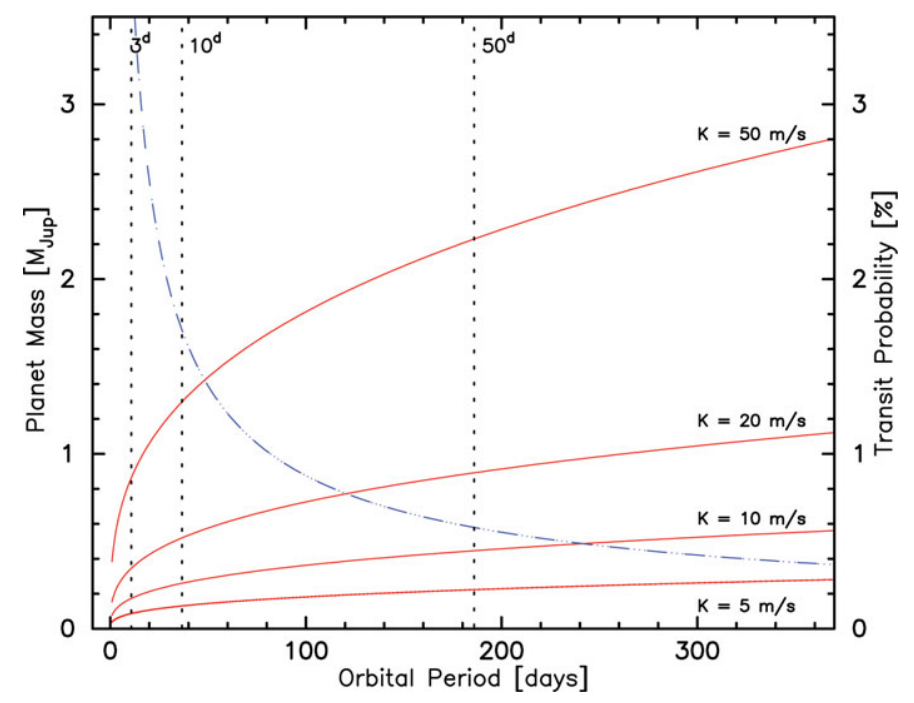

Figure 8. The same as Fig. 7 but for a $1.0+1.0 \mathrm{M}_{\odot}$ binary.

planetary periods due to the dynamical stability of their orbits (Figs. 7-8, dotted lines; see Holman \& Wiegert 1999).

The method can also be used to spectroscopically confirm transiting planet candidates around eclipsing binary stars. These may not be as hard to detect as it seems if the planetary orbit happens to be coplanar with the orbit of its parent binary star (see also Ofir 2008 in this volume) and should provide constraints on the formation of planets around close binary stars. The extensive databases of light curves from ground-based and space-based efforts and aimed at finding transiting planets may soon enable us to verify whether there exist transiting circumbinary planets around eclipsing binary stars. As shown in Figs. 7-8, the current RV precision of our method is perfectly sufficient to confirm a transiting circumbinary planet for a wide range of mass and period.

\section{Acknowledgements}

M.K. is supported by the Foundation for Polish Science through a FOCUS grant and fellowship, by the Polish Ministry of Science and Higher Education through grants N203 $00532 / 0449$ and 1P03D-021-29, and by NASA through grant NNG04GM62G. The RV search for circumbinary planets is carried out in collaboration with Matt Muterspaugh (UC Berkeley).

\section{References}

Bagnuolo, W. G., Jr. \& Gies, D. R. 1991, APJ, 376, 266

Holman, M. J. \& Wiegert, P. A. 1999, AJ, 117, 621

Konacki, M. 2005, APJ, 626, 431

Kurucz, R. L. 1995, ASP Conf. Ser. 78: Astrophysical Applications of Powerful New Databases, 205

Marcy, G. W. \& Butler, R. P. 1992, PASP, 104, 270

Zucker, S. \& Mazeh, T. 1994, APJ, 420, 806 